\title{
Symmetry and specializability in continued fractions
}

\author{
by \\ Henry Cohn (Cambridge, Mass.)
}

1. Introduction. A number defined by a series does not in general have an interesting continued fraction expansion. There are, however, some exceptions, such as the series

$$
\sum_{n=0}^{\infty} \frac{1}{2^{2^{n}}}=[0,1,4,2,4,4,6,4,2,4,6,2,4,6,4,4,2, \ldots],
$$

dealt with (independently) in [4] and [11]. The continued fraction expansion for this series has a type of symmetry known as folding symmetry. In this paper, we generalize folding symmetry, and give examples such as

$$
\begin{aligned}
\sum_{n=0}^{\infty} \frac{1}{T_{4^{n}}(2)}= & {[0,1,1,23,1,2,1,18815,3,1,23,3,1,23,1,2,1,} \\
& 106597754640383,3,1,23,1,3,23,1,3,18815,1,2,1,23, \\
& 3,1,23,1,2,1,18815,3,1,23,3,1,23,1,2,1, \ldots],
\end{aligned}
$$

where $T_{l}(x)$ is the $l$ th Chebyshev polynomial.

In addition, we prove a general characterization of this sort of series. These series are of the form

$$
\sum_{n=0}^{\infty} \frac{1}{f^{n}(m)}
$$

where $m \in \mathbb{Z}, f(x) \in \mathbb{Z}[x]$, and $f^{n}(x)$ denotes the $n$th iterate of $f(x)$. (For example, in the first case $f(x)=x^{2}$, and in the second $f(x)=T_{4}(x)$.) In addition, they are specialized, in the terminology of [10]. For example, the first is a special case of

$$
\sum_{n=0}^{\infty} \frac{1}{x^{2^{n}}}=[0, x-1, x+2, x, x, x-2, x, x+2, x, x-2, x+2, \ldots],
$$

with $x=2$. A continued fraction over $\mathbb{Q}(x)$, such as this one, with the property that each partial quotient has integer coefficients, is called specializable, because when one specializes by choosing an integer value for $x$, one 
gets immediately a continued fraction whose partial quotients are integers. The continued fraction one obtains is then called specialized. We prove a theorem (Theorem 7.12) that determines all sums of the form

$$
\sum_{n=0}^{\infty} \frac{1}{f^{n}(x)}
$$

(for $f(x) \in \mathbb{Z}[x]$ ) that have specializable continued fractions.

Before proceeding any further, we quickly review the basics of continued fractions, and give a short account of folding symmetry. Consider the continued fraction

$$
\left[a_{0}, a_{1}, \ldots, a_{n}\right]=a_{0}+\frac{1}{a_{1}+\frac{1}{a_{2}+\frac{1}{\cdots+\frac{1}{a_{n-1}+\frac{1}{a_{n}}}}}} .
$$

We define $p_{0}=a_{0}, q_{0}=1, p_{1}=a_{0} a_{1}+1, q_{1}=a_{1}$, and for $n \geq 2$,

$$
p_{n}=a_{n} p_{n-1}+p_{n-2} \quad \text { and } \quad q_{n}=a_{n} q_{n-1}+q_{n-2} .
$$

Proposition 1.1. For each $n$,

$$
\frac{p_{n}}{q_{n}}=\left[a_{0}, a_{1}, \ldots, a_{n}\right]
$$

and we have $p_{n} q_{n-1}-q_{n} p_{n-1}=(-1)^{n-1}$.

For a proof of this standard result, see Theorems 149 and 150 of [3]. Now consider the function $f_{n}(z)$ of $z$ defined by

$$
f_{n}(z)=a_{0}+\frac{1}{a_{1}+\frac{1}{a_{2}+\frac{1}{\cdots+\frac{1}{a_{n}+\frac{1}{z}}}} .}
$$

This function will be useful shortly. By Proposition 1.1,

$$
f_{n}(z)=\frac{p_{n} z+p_{n-1}}{q_{n} z+q_{n-1}} .
$$

We now define the notation we will use to talk about symmetry in continued fractions. Let $\vec{w}_{n}$ denote the word $a_{1}, a_{2}, \ldots, a_{n}$. We use $\overleftarrow{w}_{n}$ to denote 
the word $a_{n}, a_{n-1}, \ldots, a_{1}$, and $-\overleftarrow{w}_{n}$ to denote $-a_{n},-a_{n-1}, \ldots,-a_{1}$. This notation will be used mainly in continued fractions. (This is the notation of [9], where Proposition 1.2 was first stated in this form.)

We now give a proof of Proposition 1.2, known as the Folding Lemma, for completeness and in preparation for the proof in Section 4 of a generalization.

\section{Proposition 1.2.}

$$
\frac{p_{n}}{q_{n}}+\frac{(-1)^{n}}{x q_{n}^{2}}=\left[a_{0}, \vec{w}_{n}, x,-\overleftarrow{w}_{n}\right]
$$

Proof. First, note that $f_{n}^{-1}(z)=\left[0,-a_{n},-a_{n-1}, \ldots,-a_{0}+z\right]$. In particular, $f_{n}^{-1}(\infty)=\left[0,-\overleftarrow{w}_{n}\right]$. However, it is easy to see that

$$
f_{n}^{-1}(z)=\frac{-q_{n-1} z+p_{n-1}}{q_{n} z-p_{n}} .
$$

Hence, $\left[x,-\overleftarrow{w}_{n}\right]=x-q_{n-1} / q_{n}$, and

$$
\begin{aligned}
{\left[a_{0}, \vec{w}_{n}, x,-\overleftarrow{w}_{n}\right] } & =\frac{p_{n}\left(x-q_{n-1} / q_{n}\right)+p_{n-1}}{q_{n}\left(x-q_{n-1} / q_{n}\right)+q_{n-1}} \\
& =\frac{p_{n} q_{n} x-\left(p_{n} q_{n-1}-q_{n} p_{n-1}\right)}{q_{n}^{2} x}
\end{aligned}
$$

The desired result follows immediately.

A continued fraction $\left[a_{0}, \vec{w}_{n}, x_{1},-\overleftarrow{w}_{n}\right]$ is said to be folded. We generalize this notion as follows. A folded continued fraction has 2-fold symmetry. We say that $\left[a_{0}, \vec{w}_{n}, x_{1},-\overleftarrow{w}_{n}, x_{2}, \vec{w}_{n}\right]$ has 3 -fold symmetry, $\left[a_{0}, \vec{w}_{n}, x_{1}\right.$, $\left.-\overleftarrow{w}_{n}, x_{2}, \vec{w}_{n}, x_{3},-\overleftarrow{w}_{n}\right]$ has 4-fold symmetry, etc. The Folding Lemma generalizes nicely to $k$-fold symmetry. We deal with the generalization in Section 4.

For a more involved discussion of the Folding Lemma, see [9]. Folded continued fractions were discovered independently by Kmošek and Shallit. See $[4,11,12]$. The Folding Lemma first appears in $[6$, p. 209], and more explicitly in [1, p. 332].

2. Applications of the Folding Lemma. As an example of the sort of series with which we will deal in Section 6, we discuss an example due to Shallit (see [12]). This will serve as preparation for applying similar techniques later. In addition, we will need the last result of this section.

Folded continued fractions were originally studied to explain the simple continued fractions of certain series. For example, the Liouville number 
$\sum_{n=0}^{\infty} 1 / 10^{n !}$ has an amazing continued fraction expansion:

$$
\begin{gathered}
\sum_{n=0}^{\infty} \frac{1}{10^{n !}}=[0,4,1,3,5,99,1,4,3,1,4,999999999999,1,3,1, \\
3,4,1,99,5,3,1,4, \ldots] .
\end{gathered}
$$

The Folding Lemma applies to the partial sums, giving a continued fraction expansion for each of them in terms of that of the one before. For example, we easily get

$$
\begin{gathered}
\sum_{n=0}^{4} \frac{1}{10^{n !}}=[0,5,-4,-5,-100,5,4,-5,-1000000000000,5,-4,-5,100, \\
5,4,-5] .
\end{gathered}
$$

This gives the nearest integer continued fraction for the series, but it would be more interesting to get the simple continued fraction. (In a simple continued fraction, all of the partial quotients are integers, and all are positive, except perhaps the first.)

In fact, we can easily get the simple continued fraction expansion of any number with $k$-fold symmetry, assuming that $\left[a_{0}, \vec{w}_{n}\right]$ is a simple continued fraction. First, note that since $[\ldots, a, 0, b, \ldots]=[\ldots, a+b, \ldots]$, it is never a problem to have 0 occur in a continued fraction. We can apply $[\ldots, a,-\beta]=$ $[\ldots, a-1,1, \beta-1]$ to get rid of negatives. (This is the method of [10].) For example, $\left[a_{0}, \vec{w}_{n}, x_{1},-\overleftarrow{w}_{n}\right]=\left[a_{0}, a_{1}, \ldots, a_{n}, x_{1}-1,1, a_{n}-1, a_{n-1}, \ldots, a_{1}\right]$. Similarly, in the case of 3 -fold symmetry we get $\left[a_{0}, a_{1}, \ldots, a_{n}, x_{1}-1,1, a_{n}-\right.$ $\left.1, a_{n-1}, \ldots, a_{2}, a_{1}-1,1, x_{2}-1, a_{1}, \ldots, a_{n}\right]$. Note also that changing the sign of some $x_{i}$ is easily handled, since $[\ldots, a,-x-1,1, a-1, \ldots]=[\ldots, a-$ $1,1, x-1, a, \ldots]$. (Note that one can remove negatives from any continued fraction, not just one with $k$-fold symmetry.)

In this way, we get the simple continued fraction for the sum. This explains the amazing continued fraction noted above. Since the continued fraction expansion of each partial sum arises from that of the previous partial sum by an application of the Folding Lemma, we say that the continued fraction has iterated 2-fold symmetry. Iterated $k$-fold symmetry is defined analogously.

Note that the Folding Lemma applies not only to $\mathbb{Q}$, but also to $\mathbb{Q}(x)$. If $l$ is an integer greater than 1 , then the series $\sum_{n=0}^{\infty} 1 / x^{l^{n}}$ converges to a formal Laurent series. The Folding Lemma shows that it has iterated 2-fold symmetry in its continued fraction.

In addition, the partial quotients are in $\mathbb{Z}[x]$. In the terminology of [10], the continued fraction is specializable. We can specialize $x$ to any positive integer greater than 1, and get a simple continued fraction with iterated 2fold symmetry. In a typical continued fraction with polynomial coefficients, the partial quotients will have non-integral coefficients, so we will not get 
integral partial quotients if we attempt to specialize. (This point of view was first taken in [7], although these series had been studied earlier.)

Now consider $f(x) \in \mathbb{Z}[x]$ with degree greater than 1 , such that $f(x) \equiv 0$ $\left(\bmod x^{2}\right)$. If we define the iterates $f^{0}(x)=x$, and $f^{n}(x)=f\left(f^{n-1}(x)\right)$, then the Folding Lemma implies that $\sum_{n=0}^{\infty} 1 / f^{n}(x)$ has a specializable continued fraction with iterated 2-fold symmetry. In Section 7 , we will determine all polynomials $f(x) \in \mathbb{Z}[x]$ for which this sum is specializable.

3. Modified continuants. To state the Generalized Folding Lemma, we will need to use continuant polynomials. Ours will sometimes have to be modified, though, to have a sign alternation. Fix $\varepsilon= \pm 1$. We define modified continuants $K^{\prime}$ by

(1) $K^{\prime}\left(x_{1}\right)=x_{1}$.

(2) $K^{\prime}\left(x_{1}, x_{2}\right)=x_{1} x_{2}+\varepsilon$.

(3) For each $k \geq 2$,

$$
K^{\prime}\left(x_{1}, \ldots, x_{k}\right)=K^{\prime}\left(x_{1}, \ldots, x_{k-1}\right) x_{k}+\varepsilon K^{\prime}\left(x_{1}, \ldots, x_{k-2}\right) .
$$

It is sometimes convenient to define $K^{\prime}$ without any variables as $K^{\prime}()=$ 1. Note that when $\varepsilon=1$, these polynomials are the usual continuants; when $\varepsilon=-1$, they are the same except for the sign alternation.

Because modified continuants are the same as ordinary continuants, except for the sign alternation when $\varepsilon=-1$, one would expect the theory of modified continuants to be nearly the same as that of continuants, and indeed it is. For the theory of continuants, see [2]. All of our results for modified continuants are based directly on analogous results for continuants given in [2].

One fundamental fact about modified continuants is that $K^{\prime}\left(x_{1}, \ldots, x_{k}\right)$ is the sum of the terms which can be obtained by replacing each of a collection of disjoint pairs of consecutive variables in $x_{1} \ldots x_{k}$ with $\varepsilon$. This is easily seen from the definition. From this fact, it follows that the modified continuants also satisfy the recurrence relation

$$
K^{\prime}\left(x_{1}, \ldots, x_{k}\right)=x_{1} K^{\prime}\left(x_{2}, \ldots, x_{k}\right)+\varepsilon K^{\prime}\left(x_{3}, \ldots, x_{k}\right) .
$$

LEMMA 3.1. We have the continued fraction expansion

$$
\frac{K^{\prime}\left(x_{1}, \ldots, x_{m}\right)}{K^{\prime}\left(x_{2}, \ldots, x_{m}\right)}=x_{1}+\frac{\varepsilon}{x_{2}+\frac{\varepsilon}{x_{3}+\frac{\varepsilon}{\cdots+\frac{\varepsilon}{x_{m-1}+\frac{\varepsilon}{x_{m}}}}}} .
$$


Proof. The cases in which $m \leq 3$ are trivial. Now, we continue by induction on $m$. The continued fraction on the right is equal to

$$
\frac{K^{\prime}\left(x_{1}, \ldots, x_{m-1}+\varepsilon / x_{m}\right)}{K^{\prime}\left(x_{2}, \ldots, x_{m-1}+\varepsilon / x_{m}\right)} .
$$

Applying the recurrence defining $K^{\prime}$ twice gives

$$
\frac{K^{\prime}\left(x_{1}, \ldots, x_{m-2}\right)\left(x_{m-1}+\varepsilon / x_{m}\right)+\varepsilon K^{\prime}\left(x_{1}, \ldots, x_{m-3}\right)}{K^{\prime}\left(x_{2}, \ldots, x_{m-2}\right)\left(x_{m-1}+\varepsilon / x_{m}\right)+\varepsilon K^{\prime}\left(x_{2}, \ldots, x_{m-3}\right)},
$$

and then

$$
\frac{K^{\prime}\left(x_{1}, \ldots, x_{m-1}\right)+\varepsilon K^{\prime}\left(x_{1}, \ldots, x_{m-2}\right) / x_{m}}{K^{\prime}\left(x_{2}, \ldots, x_{m-1}\right)+\varepsilon K^{\prime}\left(x_{2}, \ldots, x_{m-2}\right) / x_{m}} .
$$

Multiplying the numerator and denominator by $x_{m}$ and applying the recurrence again proves the lemma.

4. The Generalized Folding Lemma. Define $S_{2}=\left[a_{0}, \vec{w}_{n}, x_{1}\right.$, $\left.-\overleftarrow{w}_{n}\right], S_{3}=\left[a_{0}, \vec{w}_{n}, x_{1},-\overleftarrow{w}_{n}, x_{2}, \vec{w}_{n}\right]$, etc. Thus, $S_{k}$ is the general form of a continued fraction with $k$-fold symmetry. Also, from now on set $\varepsilon=(-1)^{n}$ (for use in modified continuants), and for convenience set $p=p_{n}$ and $q=q_{n}$. Our principal result about $k$-fold symmetry is the following theorem, the Generalized Folding Lemma:

THEOREM 4.1. For all $k \geq 2$,

$$
S_{k}=\frac{p}{q}+\frac{(-1)^{n} K^{\prime}\left(x_{2} q, \ldots, x_{k-1} q\right)}{q K^{\prime}\left(x_{1} q, \ldots, x_{k-1} q\right)} .
$$

Proof. We prove this by induction. It is easy to check that the case $k=2$ is the Folding Lemma. Now suppose that it holds for $k-1$.

In the continued fraction by which $S_{k}$ is defined, if one omits the initial $a_{0}, \vec{w}_{n}$, then one is left with a continued fraction with $(k-1)$-fold symmetry. Call it $S_{k}^{\prime}$. As in the proof of the Folding Lemma, we know that $\left[x_{1},-\overleftarrow{w}_{n}\right]=$ $x_{1}-q_{n-1} / q_{n}$. It follows by induction that

$$
S_{k}^{\prime}=x_{1}-\frac{q_{n-1}}{q_{n}}+\frac{(-1)^{n} K^{\prime}\left(x_{3} q_{n}, \ldots, x_{k-1} q_{n}\right)}{q_{n} K^{\prime}\left(x_{2} q_{n}, \ldots, x_{k-1} q_{n}\right)} .
$$

Now, since $S_{k}=\left[a_{0}, \vec{w}_{n}, S_{k}^{\prime}\right]$, we see that

$$
S_{k}=\frac{p_{n}\left(x_{1}-\frac{q_{n-1}}{q_{n}}+\frac{(-1)^{n} K^{\prime}\left(x_{3} q_{n}, \ldots, x_{k-1} q_{n}\right)}{q_{n} K^{\prime}\left(x_{2} q_{n}, \ldots, x_{k-1} q_{n}\right)}\right)+p_{n-1}}{q_{n}\left(x_{1}-\frac{q_{n-1}}{q_{n}}+\frac{(-1)^{n} K^{\prime}\left(x_{3} q_{n}, \ldots, x_{k-1} q_{n}\right)}{q_{n} K^{\prime}\left(x_{2} q_{n}, \ldots, x_{k-1} q_{n}\right)}\right)+q_{n-1}} .
$$


This is equal to (after applying $p_{n} q_{n-1}-q_{n} p_{n-1}=(-1)^{n-1}$ )

$$
\begin{aligned}
& \frac{p q x_{1} K^{\prime}\left(x_{2} q, \ldots, x_{k-1} q\right)+(-1)^{n} p K^{\prime}\left(x_{3} q, \ldots, x_{k-1} q\right)}{q^{2} x_{1} K^{\prime}\left(x_{2} q, \ldots, x_{k-1} q\right)+(-1)^{n} q K^{\prime}\left(x_{3} q, \ldots, x_{k-1} q\right)} \\
& \quad+\frac{(-1)^{n} K^{\prime}\left(x_{2} q, \ldots, x_{k-1} q\right)}{q^{2} x_{1} K^{\prime}\left(x_{2} q, \ldots, x_{k-1} q\right)+(-1)^{n} q K^{\prime}\left(x_{3} q, \ldots, x_{k-1} q\right)},
\end{aligned}
$$

which simplifies to

$$
\frac{p}{q}+\frac{(-1)^{n} K^{\prime}\left(x_{2} q, \ldots, x_{k-1} q\right)}{q^{2} x_{1} K^{\prime}\left(x_{2} q, \ldots, x_{k-1} q\right)+(-1)^{n} q K^{\prime}\left(x_{3} q, \ldots, x_{k-1} q\right)} .
$$

Now applying (3.1) to the denominator yields the final result.

If we apply Lemma 3.1 to $S_{k}$, we arrive at the following corollary to Theorem 4.1:

COROLlary 4.2. We have the continued fraction expansion

$$
S_{k}=\frac{p}{q}+\frac{1}{q} \frac{(-1)^{n}}{x_{1} q+\frac{(-1)^{n}}{x_{2} q+\frac{(-1)^{n}}{\cdots+\frac{(-1)^{n}}{x_{k-2} q+\frac{(-1)^{n}}{x_{k-1} q}}}} .}
$$

5. Further results on $k$-fold symmetry. A few simple algebraic manipulations give the following equivalent formula for $S_{k}$ :

$$
S_{k}=\frac{p}{q}+\frac{(-1)^{n}}{x_{1} q^{2}} \frac{1}{1+\frac{(-1)^{n}}{x_{1} x_{2} q^{2}} \frac{1}{1+\frac{(-1)^{n}}{x_{2} x_{3} q^{2}} \frac{1}{\cdots+\frac{(-1)^{n}}{x_{k-2} x_{k-1} q^{2}}}}} .
$$

This expansion seems messier and less natural than that given by Corollary 4.2 , but it has one advantage. Set

$$
X_{k}=\frac{(-1)^{n} K^{\prime}\left(x_{3} q, \ldots, x_{k-1} q\right)}{q K^{\prime}\left(x_{2} q, \ldots, x_{k-1} q\right)} .
$$

The expansion above makes it clear that

$$
S_{k}=\frac{p}{q}+\frac{(-1)^{n}}{x_{1} q^{2}} \sum_{i=0}^{\infty}\left(-\frac{X_{k}}{x_{1}}\right)^{i} .
$$

One might hope that the partial sums would have interesting continued fraction expansions. In the case of 3-fold symmetry, there is a simple and 
useful description of the continued fractions for the partial sums. We have

$$
X_{3}=\frac{(-1)^{n} K^{\prime}()}{q K^{\prime}\left(x_{2} q\right)}=\frac{(-1)^{n}}{x_{2} q^{2}} .
$$

Note that $1 / X_{3}$ is an integer (assuming that $x_{2}$ is an integer). The partial sums have 4 -fold symmetry, as we see from the following proposition:

Proposition 5.1. For all $j \geq 1$, if

$$
x_{3}=-x_{1}\left(\frac{1-\left(-x_{1} / X_{3}\right)^{j}}{1-\left(-x_{1} / X_{3}\right)}\right),
$$

then

$$
\frac{p}{q}+\frac{(-1)^{n}}{x_{1} q^{2}} \sum_{i=0}^{j}\left(-\frac{X_{3}}{x_{1}}\right)^{i}=\frac{(-1)^{n} K^{\prime}\left(x_{2} q, x_{3} q\right)}{q K^{\prime}\left(x_{1} q, x_{2} q, x_{3} q\right)} .
$$

Proof. We can and shall solve the equation for the value of $x_{3}$ that makes it true. The important point is that $x_{3}$ turns out to be an integer, so 4 -fold symmetry occurs.

We now solve the equation. It is equivalent to

$$
\sum_{i=0}^{j}\left(-\frac{X_{3}}{x_{1}}\right)^{i}=\frac{1}{1+\frac{(-1)^{n}}{x_{1} x_{2} q^{2}} \frac{1}{1+\frac{(-1)^{n}}{x_{2} x_{3} q^{2}}}} .
$$

Equivalently,

$$
\frac{-X_{3} / x_{1}-\left(-X_{3} / x_{1}\right)^{j+1}}{\left(-X_{3} / x_{1}\right)^{j+1}-1}=\frac{(-1)^{n}}{x_{1} x_{2} q^{2}} \frac{1}{1+\frac{(-1)^{n}}{x_{2} x_{3} q^{2}}} .
$$

This is the same as

$$
\frac{-1+\left(-X_{3} / x_{1}\right)^{j}}{\left(-X_{3} / x_{1}\right)^{j+1}-1}=\frac{1}{1+\frac{(-1)^{n}}{x_{2} x_{3} q^{2}}} .
$$

Finally, this is equivalent to

$$
\frac{\left(-X_{3} / x_{1}\right)^{j}-1}{\left(-X_{3} / x_{1}\right)^{j+1}-\left(-X_{3} / x_{1}\right)^{j}}=\frac{x_{2} x_{3} q^{2}}{(-1)^{n}} .
$$

The formula for $x_{3}$ follows immediately.

This proposition may seem complicated and perhaps uninteresting. However, in Section 6 we will use it to explain an interesting continued fraction expansion. 
6. Applications of the Generalized Folding Lemma. We now use our results to determine some explicit continued fractions. Note that setting $k=3$ in Theorem 4.1, and possibly changing signs, shows that

$$
\frac{p}{q} \pm \frac{a}{a b q^{2} \pm 1}
$$

has 3-fold symmetry for all $a$ and $b$, and all combinations of signs. Of course, this holds true not only in the rationals, but also in fields of rational functions, for example. As before, we will work in $\mathbb{Q}(x)$ (and also its completion $\mathbb{Q}((x))$, the field of formal Laurent series).

Now suppose that $f(x)$ is polynomial over $\mathbb{Z}$ of degree greater than 1 such that $f(x) \equiv 1\left(\bmod x^{2}(x-1)\right)$ in $\mathbb{Z}[x]$. As before, define the iterates $f^{0}(x)=x, f^{n}(x)=f\left(f^{n-1}(x)\right)$. Then a trivial induction implies that

$$
f^{n}(x) \equiv 1\left(\bmod \left(f^{0}(x) f^{1}(x) \ldots f^{n-1}(x)\right)^{2}\right) .
$$

Using this, we see that the series $\sum_{n=0}^{\infty} 1 / f^{n}(x)$ (which converges to a formal Laurent series in $x$ ) has iterated 3-fold symmetry in its continued fraction expansion, with partial quotients in $\mathbb{Z}[x]$. (Similarly, there is also 3 -fold symmetry when $f(x) \equiv-1\left(\bmod x^{2}(x+1)\right)$.)

In particular, if $l$ is a non-zero multiple of 4 , then the Chebyshev polynomial $T_{l}(x)$ (defined by $T_{l}(x)=\cos \left(l \cos ^{-1}(x)\right)$ ) satisfies the congruence condition (and is of degree greater than 1). Since for all $a$ and $b, T_{a b}(x)=$ $T_{a}\left(T_{b}(x)\right)$, it follows that

$$
\sum_{n=0}^{\infty} \frac{1}{T_{l^{n}}(x)}
$$

has iterated 3-fold symmetry. Also, the partial quotients have integer coefficients, so the continued fraction is specializable. When we specialize $x$ to any positive integer greater than 1 , we get a simple continued fraction with iterated 3-fold symmetry. For example,

$$
\begin{aligned}
\sum_{n=0}^{\infty} \frac{1}{T_{4^{n}}(2)}= & {[0,1,1,23,1,2,1,18815,3,1,23,3,1,23,1,2,1,} \\
& 106597754640383,3,1,23,1,3,23,1,3,18815,1,2,1,23, \\
& 3,1,23,1,2,1,18815,3,1,23,3,1,23,1,2,1, \ldots] .
\end{aligned}
$$

When $l$ is not divisible by 4 , the series (6.1) displays more complicated behavior. Here is an apparently typical example of the case when $l$ is odd:

$$
\begin{aligned}
\sum_{n=0}^{\infty} \frac{1}{T_{3^{n}}(2)}= & {[0,1,1,5,1,414,1,2,4,280903,1,3,3,207,2,5,1,} \\
& 22165307996832415,6,2,207,3,4,140451,1,3,3, \\
& 118,2,2,1,1,7, \ldots] .
\end{aligned}
$$


There is partial symmetry, but it breaks down. It would be interesting to have an explanation of this behavior, and of that for $l \equiv 2(\bmod 4)$. (By Theorem 7.12, these series never have specializable continued fractions.)

We can also use Proposition 5.1 to prove that certain series have 4-fold symmetry in their continued fractions. For example, Proposition 5.1 implies that for each $l$,

$$
\sum_{n=0}^{3 l+2} \frac{1}{x^{2^{n}}}+\sum_{n=0}^{l} \frac{1}{x^{3 \cdot 8^{n}}}
$$

has iterated 4 -fold symmetry. To see this, write the sum as

$$
\sum_{n=0}^{l}\left(\frac{1}{x^{8^{n}}}+\frac{1}{x^{2 \cdot 8^{n}}}+\frac{1}{x^{3 \cdot 8^{n}}}+\frac{1}{x^{4 \cdot 8^{n}}}\right)
$$

and apply Proposition 5.1 with $q=x^{4 \cdot 8^{l-1}}, j=3, x_{1}=(-1)^{n}$, and $x_{2}=-1$. This explains the symmetry observed in

$$
\begin{aligned}
\sum_{n=0}^{\infty} \frac{1}{2^{2^{n}}}+\sum_{n=0}^{\infty} \frac{1}{8^{8^{n}}}= & {[0,1,16,14,16,1,65792,15,17,65792} \\
& 1,16,14,16,18,14,16,1,65792,16 \\
& 340282366920938463481821351505477763072 \\
& 1,15,65792,1,16,14,16, \ldots]
\end{aligned}
$$

Also, one checks easily that the extremely large partial quotients are exactly the numbers $2^{2^{3 n+1}}+2^{2^{3 n}}$.

One can generate other examples of this phenomenon. For example, Proposition 5.1 implies that

$$
\sum_{n=0}^{\infty} \frac{1}{x^{6^{n}}}+\sum_{n=0}^{\infty} \frac{1}{x^{2 \cdot 6^{n}}}+\sum_{n=0}^{\infty} \frac{1}{x^{3 \cdot 6^{n}}}
$$

has iterated 4 -fold symmetry. It is unclear whether it is essentially a coincidence that these sums have iterated 4 -fold symmetry, or whether there is some more general result along these lines.

7. Specializability. These results bring up some interesting related questions. Suppose that $f(x)$ is a polynomial over $\mathbb{Z}$, of degree greater than 1. Under what conditions does

$$
\sum_{n=0}^{\infty} \frac{1}{f^{n}(x)}
$$

have a specializable continued fraction? Several of the sums dealt with earlier in this paper were of this form, with the allowable functions characterized by congruence conditions. In this section, we will show that in general, such 
a series is specializable if and only if $f(x)$ satisfies one of fourteen congruence conditions.

All of our examples so far have had symmetry. However, this is not the case in general. We will prove shortly that if $f(x) \equiv-x\left(\bmod x^{2}\right)$, then the sum has a specializable continued fraction, although $k$-fold symmetry does not occur. The proof is fairly typical of our methods in this section. We first guess what the continued fraction expansion is, and then prove it by induction. Surprisingly, this works in every case in which the continued fraction expansion is specializable, although several cases are tricky. More surprisingly, we can actually rule out every other case, and thereby arrive at a complete classification of the polynomials for which the series (7.1) has a specializable continued fraction.

Before we deal with the case of $f(x) \equiv-x\left(\bmod x^{2}\right)$, we need to set up some notation. Let $f(x)=g(x) x^{2}-x$, and then set $A_{1}(x)=-g(x), A_{2}(x)=$ $g(f(x)) x^{2}, A_{3}(x)=-g\left(f^{2}(x)\right)(f(x) / x)^{2}, A_{4}(x)=g\left(f^{3}(x)\right) x^{2}\left(f^{2}(x) / f(x)\right)^{2}$, etc. In general,

$$
\begin{array}{r}
A_{2 l}(x)=g\left(f^{2 l-1}(x)\right) x^{2}\left(f^{2}(x) / f(x)\right)^{2}\left(f^{4}(x) / f^{3}(x)\right)^{2} \ldots \\
\ldots\left(f^{2 l-2}(x) / f^{2 l-3}(x)\right)^{2},
\end{array}
$$

and

$$
A_{2 l+1}(x)=-g\left(f^{2 l}(x)\right)(f(x) / x)^{2}\left(f^{3}(x) / f^{2}(x)\right)^{2} \ldots\left(f^{2 l-1}(x) / f^{2 l-2}(x)\right)^{2} .
$$

(The condition on $f(x)$ implies that these are polynomials.) Then we have the following result:

Proposition 7.1. Let $f(x)=g(x) x^{2}-x$, and $A_{i}(x)$ be as defined above. Then for each $l$,

$$
\sum_{n=0}^{l} \frac{1}{f^{n}(x)}=\left[0, x, A_{1}(x), A_{2}(x), \ldots, A_{l}(x)\right] .
$$

Proof. We prove this by induction. The base case is trivial. Now note that

$$
-x^{2}\left[A_{1}(f(x)), A_{2}(f(x)), \ldots, A_{l}(f(x))\right]=\left[A_{2}(x), A_{3}(x), \ldots, A_{l+1}(x)\right] .
$$

This, combined with the identity $\left[0, x,-g(x),-x^{2} X\right]=1 / x+[0, f(x), X]$, proves the result.

We get a similar result when $f(x) \equiv x^{2}-x+1\left(\bmod x^{2}(x-1)^{2}\right)$. Let $f(x)=x^{2}-x+1+x^{2}(x-1)^{2} g(x)$, and set $A_{1}(x)=-g(x)(x-1)^{2}, B_{1}(x)=$ $-x^{2}$, and in general

$$
A_{l}(x)=-g\left(f^{l-1}(x)\right)\left(\frac{f^{l-1}(x)-1}{x f(x) \ldots f^{l-2}(x)}\right)^{2}
$$


and

$$
B_{l}(x)=-x^{2} f(x)^{2} f^{2}(x)^{2} \ldots f^{l-1}(x)^{2} .
$$

(As in the previous example, the condition on $f(x)$ implies that these are polynomials.) Then we have the following result:

Proposition 7.2. Let $f(x)=x^{2}-x+1+x^{2}(x-1)^{2} g(x)$, and $A_{i}(x)$ and $B_{i}(x)$ be as defined above. Then for each $l$,

$$
\sum_{n=0}^{l} \frac{1}{f^{n}(x)}=\left[0, x, A_{1}(x)-1, B_{1}(x), A_{2}(x), B_{2}(x), \ldots, A_{l}(x), B_{l}(x)\right] .
$$

Pro of. We prove this by induction. The base case is trivial. Now note that

$$
\begin{aligned}
{\left[A_{1}(f(x)), B_{1}(f(x)), \ldots, A_{l}(f(x))\right.} & \left., B_{l}(f(x))\right] \\
& =x^{2}\left[A_{2}(x), B_{2}(x), \ldots, A_{l+1}(x), B_{l+1}(x)\right] .
\end{aligned}
$$

This, combined with the identity $\left[0, x, A_{1}(x)-1, B_{1}(x), X\right]=1 / x+[0, f(x)$, $\left.-1+x^{2} X\right]$, proves the result.

Note that in the special case where $f(x)=x^{2}-x+1$, we have

$$
\sum_{n=0}^{\infty} \frac{1}{f^{n}(x)}=\frac{1}{x-1}
$$

Of course, this example is rather special.

We now determine exactly which polynomials $f(x)$ are such that all of the partial sums of $\sum_{n=0}^{\infty} 1 / f^{n}(x)$ have specializable continued fractions. Before doing this, however, we need to discuss a few points about proving non-specializability.

Suppose that one has a continued fraction expansion for a formal Laurent series. If one of the partial quotients does not have integral coefficients, it is not necessarily the case that the Laurent series does not have a specializable continued fraction. To see this, note that the identity $[a+1 / b, c]=\left[a, b,-(c+b) / b^{2}\right]$ shows that when the constant term of a partial quotient is not an integer, one can on occasion adjust the continued fraction to make it specializable. For example, $\left[x-1 / 3,9 x^{2}+3\right]=\left[x,-3,-x^{2}\right]$. However, it is not hard to see that if the first partial quotient without integer coefficients has a non-integral coefficient other than the constant term, then the Laurent series has no specializable continued fraction. This observation will suffice for all of the examples we will consider.

Also, we will look at continued fractions involving several variables. When one wants to prove that such a continued fraction is not specializable, one must be careful about 0 as a partial quotient. If one of the partial quotients becomes 0 for certain values of some of the variables, then its two neighboring partial quotients add when the variables assume those values. 
This could make the continued fraction specializable. In general, it will be clear that this does not happen, however.

We begin with the following easy lemma.

Lemma 7.3. Let $f(x)$ be a (non-zero) polynomial over $\mathbb{Z}$. Then $1 / x+$ $1 / f(x)$ has a specializable continued fraction iff $f(x)$ is congruent modulo $x^{2}$ to one of

$$
0,-1,1,-x,-x-1,-x+1,-2 x,-2 x-1,-2 x+1 .
$$

Proof. Suppose that $f(x)=g(x) x^{2}+b x+a$. Then

$$
\frac{1}{x}+\frac{1}{f(x)}=\left[0, x,-g(x),-\frac{x}{b+1}+\frac{a}{(b+1)^{2}},-\frac{(b+1)^{3} x}{a^{2}}-\frac{(b+1)^{2}}{a}\right] .
$$

From this, we see that unless $b=0, b=-1$, or $b=-2$, there is no specializable continued fraction for $1 / x+1 / f(x)$. Now, we just check each case.

When $b=0,1 / x+1 / f(x)=\left[0, x,-g(x),-x+a,-x / a^{2}-1 / a\right]$. When $b=-1,1 / x+1 / f(x)=\left[0, x,-g(x),-x^{2} / a\right]$. When $b=-2,1 / x+1 / f(x)=$ $\left[0, x,-g(x), x+a, x / a^{2}-1 / a\right]$. From these, we see that in each case, we must have $a=-1, a=0$, or $a=1$ to have specializability, and that in each of those cases, $1 / x+1 / f(x)$ does have a specializable continued fraction.

Lemma 7.4. Let $f(x)$ be a (non-zero) polynomial over $\mathbb{Z}$, and suppose that $f(x)=1+k x^{2}+x^{2}(x-1) g(x)$ with $g(x) \in \mathbb{Z}[x]$. Then $1 / x+1 / f(x)+1 / f^{2}(x)$ has a specializable continued fraction iff $k=0$.

Proof. We look at the more general sum

$$
\frac{1}{x}+\frac{1}{f(x)}+\frac{1}{1+k f(x)^{2}+f(x)^{2}(f(x)-1) G(x)} .
$$

(Note that we do not require that $G(x)=g(f(x))$.)

If $k=0$, this has the continued fraction expansion $[0, x,-g(x)(x-$ $1),-x+1,-x-1, G(x) g(x)(x-1), x+1, x-1, g(x)(x-1),-x+1,-x-$ $1, g(x)(x-1), x-1, x+1]$. If $k=1$, then it has the expansion [0, $x,-g(x)(x-$ $1)-1,-x+1,-x-1, G(x) g(x)(x-1)+G(x), x,-g(x) x^{3}+(g(x)-1) x^{2},-x$, $g(x)(x-1)+1, x-2, x / 4+1 / 2]$, which cannot be made specializable. Finally, for all other values of $k$, it has the expansion $[0, x,-g(x)(x-1)-$ $k,-x+1,-x-1, G(x) g(x)(x-1)+G(x) k, x+1-k, x /\left(k^{2}-2 k+1\right)+$ $1 /(k-1), g(x)\left(k^{2}-2 k+1\right)^{2} x+\left(k^{2}-2 k+1\right)\left(k-g(x)+k^{3}+2 k g(x)-2 k^{2}-\right.$ $\left.k^{2} g(x)\right),-x /\left(k^{2}-2 k+1\right)-1 /(k-1),-x-1+k, g(x)(x-1)+k, x-1-$ $\left.k, x /\left(1+2 k+k^{2}\right)+1 /(k+1)\right]$, which also cannot be made specializable.

Now note that changing $f(x)$ to $-f(-x)$ has no effect on the specializability of the continued fractions for the partial sums we are studying. Thus, we immediately get the following lemma: 
LEMma 7.5. Let $f(x)$ be a (non-zero) polynomial over $\mathbb{Z}$, and suppose that $f(x)=-1+k x^{2}+x^{2}(x+1) g(x)$ with $g(x) \in \mathbb{Z}[x]$. Then $1 / x+1 / f(x)+$ $1 / f^{2}(x)$ has a specializable continued fraction iff $k=0$.

LEMma 7.6. Let $f(x)$ be a (non-zero) polynomial over $\mathbb{Z}$, and suppose that $f(x)=-2 x+x^{2} g(x)$ with $g(x) \in \mathbb{Z}[x]$. Then $1 / x+1 / f(x)+1 / f^{2}(x)$ never has a specializable continued fraction.

Proof. As in the proof of Lemma 7.4, we generalize slightly, and look at

$$
\frac{1}{x}+\frac{1}{f(x)}+\frac{1}{-2 f(x)+f(x)^{2} G(x)},
$$

where $G(x)$ need not be equal to $g(f(x))$. This has the continued fraction expansion $[0, x,-g(x), x,-G(x),-x,-g(x), x / 3]$, which cannot be made specializable.

Similar arguments, best carried out using computer algebra software, prove the following two lemmas:

LEMMA 7.7. Let $f(x)$ be a (non-zero) polynomial over $\mathbb{Z}$, and suppose that $f(x) \equiv-2 x+1\left(\bmod x^{2}\right)$. Then $1 / x+1 / f(x)+1 / f^{2}(x)$ has a specializable continued fraction iff $f(x) \equiv-x^{3}+3 x^{2}-2 x+1\left(\bmod x^{2}(x-1)^{2}\right)$ or $f(x) \equiv$ $x^{2}-2 x+1\left(\bmod x^{2}(x-1)^{2}\right)$.

LEMMA 7.8. Let $f(x)$ be a (non-zero) polynomial over $\mathbb{Z}$, and suppose that $f(x) \equiv-x+1\left(\bmod x^{2}\right)$. Then $1 / x+1 / f(x)+1 / f^{2}(x)$ has a specializable continued fraction iff $f(x) \equiv x^{2}-x+1, x^{3}-x+1, x^{3}-x^{2}-x+1$, or $-x^{3}+2 x^{2}-x+1\left(\bmod x^{2}(x-1)^{2}\right)$.

(Of course, changing $f(x)$ to $-f(-x)$ gives analogous results for the cases in which $f(x) \equiv-2 x-1\left(\bmod x^{2}\right)$ or $f(x) \equiv-x-1\left(\bmod x^{2}\right)$.)

In fact, when $f(x) \equiv x^{3}-x+1\left(\bmod x^{2}(x-1)^{2}\right)$, one can check that $1 / x+1 / f(x)+1 / f^{2}(x)+1 / f^{3}(x)$ does not have a specializable continued fraction. This, combined with the preceding lemmas, proves the necessity of the conditions of the following theorem:

THEOREM 7.9. Let $f(x)$ be a polynomial over $\mathbb{Z}$, of degree greater than 1. All of the partial sums of $\sum_{n=0}^{\infty} 1 / f^{n}(x)$ have specializable continued fractions if and only if $f(x)$ satisfies one of the following congruences:

(1) $f(x) \equiv 0\left(\bmod x^{2}\right)$.

(2) $f(x) \equiv-x\left(\bmod x^{2}\right)$.

(3) $f(x) \equiv 1\left(\bmod x^{2}(x-1)\right)$.

(4) $f(x) \equiv-1\left(\bmod x^{2}(x+1)\right)$.

(5) $f(x) \equiv x^{3}-x^{2}-x+1\left(\bmod x^{2}(x-1)^{2}\right)$.

(6) $f(x) \equiv-x^{3}+2 x^{2}-x+1\left(\bmod x^{2}(x-1)^{2}\right)$.

(7) $f(x) \equiv-x^{3}+3 x^{2}-2 x+1\left(\bmod x^{2}(x-1)^{2}\right)$. 
(8) $f(x) \equiv x^{3}+x^{2}-x-1\left(\bmod x^{2}(x+1)^{2}\right)$.

(9) $f(x) \equiv-x^{3}-2 x^{2}-x-1\left(\bmod x^{2}(x+1)^{2}\right)$.

(10) $f(x) \equiv-x^{3}-3 x^{2}-2 x-1\left(\bmod x^{2}(x+1)^{2}\right)$.

(11) $f(x) \equiv x^{2}-x+1\left(\bmod x^{2}(x-1)^{2}\right)$.

(12) $f(x) \equiv x^{2}-2 x+1\left(\bmod x^{2}(x-1)^{2}\right)$.

(13) $f(x) \equiv-x^{2}-x-1\left(\bmod x^{2}(x+1)^{2}\right)$.

(14) $f(x) \equiv-x^{2}-2 x-1\left(\bmod x^{2}(x+1)^{2}\right)$.

Proof. We have already proved the necessity that one of these congruences hold. We now prove its sufficiency.

Because of our results so far, and the symmetry between $f(x)$ and $-f(-x)$, we see that we need only deal with (5), (6), (7), and (12). Set $S_{n}(x)=1 / x+1 / f(x)+\cdots+1 / f^{n}(x)$, and $Q_{n}(x)=x f(x) \ldots f^{n}(x)$.

We begin with (6). Suppose that $f(x)=-x^{3}+2 x^{2}-x+1+x^{2}(x-1)^{2} g(x)$. First, define the polynomials $A_{0}(x)=1, A_{1}(x)=(1-f(x)) / x$, and for $n \geq 2$

$$
A_{n}(x)=\frac{1-f^{n}(x)}{x f(x) \ldots f^{n-1}(x)}+f^{n}(x) f^{n-1}(x) A_{n-2}(x) .
$$

(Because $f(x)-1=x(x-1)^{2}(x g(x)-1)$, these are indeed polynomials. In fact, we see from this that for $n>1, f^{n}(x)-1$ is divisible by $Q_{n-1}(x) Q_{n-2}(x)$.)

In fact, $A_{n}(x)$ is the denominator of the penultimate convergent of $S_{n}(x)$. We will see later that the continued fraction expansion of $S_{n}(x)$ has even length. If $S_{n}(x)=p / q$ with $p$ and $q$ coprime, then $p^{\prime} / q^{\prime}$ is the penultimate convergent iff $p q^{\prime}-q p^{\prime}=1$, and also $\operatorname{deg} q^{\prime}<\operatorname{deg} q$ and $\operatorname{deg} p^{\prime}<\operatorname{deg} p$. In this case, it is easiest to check simply that $q^{\prime}(p / q)-1 / q$ is a polynomial of degree less than $\operatorname{deg} p=\operatorname{deg} q-1$. Of course, the denominator of $S_{n}(x)$ is easily seen to be $Q_{n}(x)$. (This is the method used in [10].)

Our proof that $A_{n}(x)$ is the denominator of the penultimate convergent will be by induction. The first two cases are easy to check. Also, note that if $S_{n}(x) A_{n}(x)-1 / Q_{n}(x)$ is a polynomial, then it automatically has the right degree. Now, we express it as

$$
\begin{aligned}
& \left(S_{n-2}(x)+\frac{1}{f^{n-1}(x)}+\frac{1}{f^{n}(x)}\right) \\
& \times\left(\frac{1-f^{n}(x)}{Q_{n-1}(x)}+f^{n}(x) f^{n-1}(x) A_{n-2}(x)\right)-\frac{1}{Q_{n}(x)} .
\end{aligned}
$$

The product $\left(1 / f^{n-1}(x)+1 / f^{n}(x)\right) f^{n}(x) f^{n-1}(x) A_{n-2}(x)$ is a polynomial, as is $S_{n-2}(x)\left(1-f^{n}(x)\right) / Q_{n-1}(x)$. (The latter one is a polynomial since $f^{n}(x)-1$ is divisible by $Q_{n-1}(x) Q_{n-2}(x)$.) Also by induction we deduce that $S_{n-2}(x) A_{n-2}(x) f^{n}(x) f^{n-1}(x)$ is a polynomial plus $f^{n}(x) f^{n-1}(x) / Q_{n-2}(x)$. 
Thus, we need only show that

$$
\frac{f^{n}(x) f^{n-1}(x)}{Q_{n-2}(x)}+\left(\frac{1}{f^{n-1}(x)}+\frac{1}{f^{n}(x)}\right)\left(\frac{1-f^{n}(x)}{Q_{n-1}(x)}\right)-\frac{1}{Q_{n}(x)}
$$

is a polynomial, or equivalently that

$$
f^{n}(x) f^{n-1}(x)+\left(\frac{1}{f^{n-1}(x)}+\frac{1}{f^{n}(x)}\right)\left(\frac{1-f^{n}(x)}{f^{n-1}(x)}\right)-\frac{1}{f^{n}(x) f^{n-1}(x)}
$$

is a polynomial which is divisible by $Q_{n-2}(x)$. This follows immediately from the fact that when $n=2$, it is a polynomial which is divisible by $f(x)-1$, because one sees then that it is always a polynomial divisible by $f^{n-1}(x)-1$, which is divisible by $Q_{n-2}(x) Q_{n-3}(x)$.

Now let $X_{1}(x)=-g(x)(x-1)^{2}+x-2$, and for $n \geq 2$,

$$
X_{n}(x)=\frac{A_{n-2}(x)}{Q_{n-2}(x)}-\frac{A_{n-1}(x)}{Q_{n-1}(x)}+\frac{1-f^{n}(x)}{Q_{n-1}(x)^{2}} .
$$

(One can prove by induction that this is a polynomial, by looking at the differences $X_{n}(x)-X_{n-2}(x)$ and showing that they are polynomials in a way like that used to prove that (7.2) is a polynomial.)

Note that $S_{1}(x)=\left[0, x, X_{1}(x),-x^{2}\right]$. Now suppose that $\vec{s}_{n}$ is such that for each $n, S_{n}(x)=\left[0, \vec{s}_{n}\right]$. Then for $n \geq 2$ we have $S_{n}(x)=\left[0, \vec{s}_{n-1}\right.$, $\left.X_{n}(x),-\overleftarrow{s}_{n-2}, f^{n-1}(x)^{2}, \vec{s}_{n-2}\right]$. (Strictly speaking, one should probably define $\vec{s}_{n}$ inductively as $\vec{s}_{n-1}, X_{n}(x),-\overleftarrow{s}_{n-2}, f^{n-1}(x)^{2}, \vec{s}_{n-2}$, and then note that $S_{n}(x)=\left[0, \vec{s}_{n}\right]$.)

This is not hard to prove. Of course,

$$
\left[X_{n}(x),-\overleftarrow{s}_{n-2}\right]=X_{n}(x)-A_{n-2}(x) / Q_{n-2}(x) .
$$

It follows from the Folding Lemma that

$$
\begin{aligned}
{\left[X_{n}(x),-\overleftarrow{s}_{n-2}, f^{n-1}(x)^{2},\right.} & \left.\vec{s}_{n-2}\right] \\
& =X_{n}(x)-\frac{A_{n-2}(x)}{Q_{n-2}(x)}-\frac{1}{f^{n-1}(x)^{2} Q_{n-2}(x)^{2}} .
\end{aligned}
$$

From this, one sees that if one subtracts $S_{n-1}(x)$ from the whole continued fraction, one gets

$$
\begin{aligned}
\left(Q _ { n - 1 } ( x ) \left(-Q_{n-1}(x)\left(X_{n}(x)\right.\right.\right. & -\frac{A_{n-2}(x)}{Q_{n-2}(x)} \\
& \left.\left.\left.-\frac{1}{f^{n-1}(x)^{2} Q_{n-2}(x)^{2}}\right)-A_{n-1}(x)\right)\right)^{-1} .
\end{aligned}
$$

If one applies the definitions of $X_{n}(x)$ and of $Q_{n}(x)$, this simplifies to $1 / f^{n}(x)$. (There is no need to use the recurrence defining $A_{l}(x)$ in these 
manipulations, because all of the occurrences of $A_{l}(x)$ (for various $l$ ) cancel.) We omit the details. This proves the sufficiency of case (6).

We now deal with case (12). Suppose that $f(x)=(x-1)^{2}\left(1+g(x) x^{2}\right)$. First, define the polynomials (for $n \geq 1$ )

$$
A_{n}(x)=\frac{f^{n}(x)-1}{f^{n-1}(x)}+f^{n}(x) f^{n-1}(x) S_{n-1}(x) .
$$

(One can check easily that these are polynomials.)

As before, $A_{n}(x)$ is the denominator of the penultimate convergent of $S_{n}(x)$. We will prove that the continued fraction expansion of $S_{n}(x)$ has odd length. Assuming this, it is easy to prove that $A_{n}(x)$ is indeed this denominator. Note that the denominator of $S_{n}(x)$ is $f^{n}(x) f^{n-1}(x)$. Let $B_{n}(x)=A_{n}(x) S_{n}(x)+1 /\left(f^{n}(x) f^{n-1}(x)\right)$. We need to show that $B_{n}(x)$ is a polynomial. To do this by induction, we look at the difference

$$
\begin{aligned}
B_{n}(x)-B_{n-1}(f(x))= & \left(A_{n-1}(f(x))+\frac{f^{n}(x) f^{n-1}(x)}{x}\right)\left(S_{n-1}(f(x))+\frac{1}{x}\right) \\
& -A_{n-1}(f(x)) S_{n-1}(f(x)),
\end{aligned}
$$

and show that it is a polynomial. It is easy to check that $f^{n}(x) f^{n-1}(x) / x^{2}$ is a polynomial. Thus, this reduces to checking that $A_{n-1}(f(x))+$ $S_{n-1}(f(x)) f^{n}(x) f^{n-1}(x)$ is a polynomial divisible by $x$. Call this $C_{n}(x)$. Then $C_{n+1}(x)=C_{n}(f(x))+f^{n}(x) f^{n-1}(x) / x+f^{n+1}(x) f^{n}(x) / f(x)$, and one sees by induction that these are polynomials divisible by $x$.

Now, for $n \geq 2$, define

$$
X_{n}(x)=g\left(f^{n-1}(x)\right)\left(\frac{f^{n-1}(x)-1}{f^{n-2}(x)}\right)^{2}
$$

and for $n \geq 3$,

$$
Y_{n}(x)=-\frac{f^{n-1}(x)}{f^{n-3}(x)^{2}} .
$$

Also define $Y_{2}(x)=f(x)$.

Now note that $S_{1}(x)=\left[0, x,-g(x)(x-1)^{2}-1, x+1, x-1\right]$ and $S_{2}(x)=$ $\left[0, x,-g(x)(x-1)^{2}-1, x+1, x-1, X_{2}(x),-x, f(x)+1, x,-g(x)(x-1)^{2}-1, x\right]$. Suppose that for each $n, S_{n}(x)=\left[0, \vec{s}_{n}\right]$. Then for $n \geq 3$, we have

$$
\begin{aligned}
S_{n}(x)=[0, & \vec{s}_{n-1}, X_{n}(x) \\
& \left.-\vec{s}_{n-2}, Y_{n}(x), \vec{s}_{n-3}, Y_{n-1}(x),-\overleftarrow{s}_{n-3}, X_{n-1}(x), \overleftarrow{s}_{n-2}\right]
\end{aligned}
$$

This can be proved completely straightforwardly. The case $n=3$ seems to need to be treated differently, since it involves $Y_{2}(x)$, which does not quite fit the pattern defining $Y_{n}(x)$ in general, but the same methods suffice to prove it. 
In the general case, the techniques we have been using show that the continued fraction in (7.3) is equal to a certain rational function of iterates of $f(x), g(x)$ applied to iterates of $f(x)$, and partial sums of the series. (Note that the numerators and denominators of the last two convergents of $S_{n}(x)$ can be expressed in terms of these.)

When one expresses each of the partial sums in terms of $S_{n}(x)$ (and the iterates of $f(x)$ ) and subtracts off $S_{n}(x)$, one is left with a rational function of the iterates of $f(x)$, and $g(x)$ applied to them. (The partial sums all disappear by cancellation.) One can then express each of the iterates of $f(x)$ in terms of the first one to appear. This gives a rational function of one iterate of $f(x)$, as well as $g(x)$ applied to several iterates. This rational function vanishes identically. (This would be tedious to check by hand, but is not very difficult to check using computer algebra software.)

Now, we deal with case (5). Suppose that $f(x)=(x-1)^{2}\left(1+x+x^{2} g(x)\right)$. Now define $A_{1}(x)=(f(x)-1) / x$, and for $n \geq 2$,

$$
A_{n}(x)=\frac{f^{n}(x)-1}{f^{n-1}(x)}+f^{n}(x) f^{n-1}(x) S_{n-2}(x) .
$$

One can check, as before, that this is the denominator of the penultimate convergent of $S_{n}(x)$. We will prove shortly that the continued fraction has odd length for $n \geq 2$. Note that the denominator of $S_{n}(x)$ is $f^{n}(x) f^{n-1}(x)$.

Now define $X_{n}(x)$ for $n \geq 3$ to be

$$
g\left(f^{n-1}(x)\right)\left(\frac{f^{n-1}(x)-1}{f^{n-2}(x)}\right)^{2}+g\left(f^{n-2}(x)\right)\left(f^{n-2}(x)-1\right)^{2}+f^{n-2}(x)-1,
$$

$Y_{n}(x)$ for $n \geq 4$ to be

$$
\begin{aligned}
& \left(\frac{f^{n-2}(x)-1}{f^{n-3}(x)}\right)^{2} \\
& \quad \times\left(g\left(f^{n-2}(x)\right)\left(f^{n-1}(x)+\left(f^{n-2}(x)+1\right)\left(f^{n-2}(x)-1\right)^{2}\right)+f^{n-2}(x)^{2}-2\right),
\end{aligned}
$$

and $Z_{n}(x)$ for $n \geq 6$ to be

$$
-Y_{n-2}(x)-\frac{f^{n-2}(x) f^{n-3}(x)^{2}}{f^{n-4}(x)^{2} f^{n-5}(x)^{2}} .
$$

(These are easily seen to be polynomials.)

Also define $X_{1}(x)=-g(x)(x-1)^{2}-x+1$, and $X_{2}(x)$ following the definition above, except multiplied by -1 . Define $Y_{1}(x)=-x^{2}, Y_{2}(x)=$ $-g(x)(x-1)^{4}\left(g(x) x^{2}+2 x+2\right)-x^{4}+2 x^{3}+x^{2}-4 x+1$, and $Y_{3}(x)$ following the definition above, except multiplied by -1 . Finally, define $Z_{3}(x)=x^{2}(f(x)+$ $1), Z_{4}(x)=\left(f(x)^{2}(f(f(x))+1)+2 x-1\right) / x^{2}$, and $Z_{5}(x)$ following the definition above, except with the second term multiplied by -1 . 
Now note that $S_{1}(x)=\left[0, x, X_{1}(x), Y_{1}(x)\right]$ and $S_{2}(x)=\left[0, x, X_{1}(x)\right.$, $\left.Y_{1}(x), X_{2}(x), x-1, x+1, Y_{2}(x), x\right]$. Suppose that for each $n, S_{n}(x)=\left[0, \vec{s}_{n}\right]$. Then for $n \geq 3$ we have

$$
\begin{aligned}
S_{n}(x)=[0, & \vec{s}_{n-1}, X_{n}(x), \\
& \left.\quad-\vec{s}_{n-2}, 0, \vec{s}_{n-4}, Y_{n-2}(x), 0, Z_{n}(x),-\overleftarrow{s}_{n-4}, Y_{n}(x), \overleftarrow{s}_{n-2}\right] .
\end{aligned}
$$

(When $n=3, \vec{s}_{-1}$ and $-\overleftarrow{s}_{-1}$ appear here. They should be interpreted to be empty words. Also, note that because 0 appears in this expansion, the continued fraction collapses somewhat. However, it remains specializable.)

As before, this is straightforward to prove. The methods used for the proof of the previous cases also work in this case. (It is best to use computer algebra software for the calculations.)

Finally, we deal with case (7). First note that given any continued fraction $\left[a_{0}, a_{1}, \ldots, a_{n}\right]$ with convergents $p_{i} / q_{i}$, and given any $X$, we have

$$
\left[a_{0}, \vec{w}_{n}, X, \overleftarrow{w}_{n}, 1,-\vec{w}_{n}\right]=\frac{p_{n}}{q_{n}}+\frac{1}{q_{n}\left(X q_{n}+2 q_{n-1}\right)(-1)^{n}+1} .
$$

(This is easy to prove using the techniques used to prove the Generalized Folding Lemma.)

Now, suppose that $f(x)=-x^{3}+3 x^{2}-2 x+1+g(x) x^{2}(x-1)^{2}$. Note that $f(x)-1=x(x-1)\left(g(x)\left(x^{2}-x\right)-x+2\right)$, and that the denominator of $S_{n}(x)$ is $Q_{n}(x)$. We will show that the continued fraction of $S_{n+1}(x)$ arises from that of $S_{n}(x)$ through application of (7.4), for a suitable choice of $X$.

To prove this, we use the fact that for any continued fraction, $p_{k} q_{k-1} \equiv$ $(-1)^{k-1}\left(\bmod q_{k}\right)$. (This follows from the equation $p_{k} q_{k-1}-q_{k} p_{k-1}=$ $(-1)^{k-1}$.) From this, one sees immediately that (7.4) is applicable (with $X$ a polynomial) if and only if

$$
S_{n}(x)\left(f^{n+1}(x)-1\right) \equiv-2\left(\bmod Q_{n}(x)\right) .
$$

(It is easy to check that $Q_{n}(x)$ divides $f^{n+1}(x)-1$, so $S_{n}(x)\left(f^{n+1}(x)-1\right)$ is a polynomial.)

However, this is easy to prove by induction. The base case is trivial. Now, assume that it holds for $n-1$. Using induction and the fact that $f^{n}(x)-1$ divides $f^{n+1}(x)-1$, we have

$$
S_{n-1}(x)\left(f^{n+1}(x)-1\right) \equiv-2\left(\frac{f^{n+1}(x)-1}{f^{n}(x)-1}\right)\left(\bmod Q_{n}(x)\right) .
$$

Hence,

$$
S_{n}(x)\left(f^{n+1}(x)-1\right) \equiv-2\left(\frac{f^{n+1}(x)-1}{f^{n}(x)-1}\right)+\frac{f^{n+1}(x)-1}{f^{n}(x)}\left(\bmod Q_{n}(x)\right) .
$$

This simplifies to $-f^{n}(x)\left(f^{n}(x)-1\right)\left(\left(f^{n}(x)+1\right) g\left(f^{n}(x)\right)-1\right)-2$. Because $Q_{n-1}(x)$ divides $f^{n}(x)-1$, this is -2 modulo $Q_{n}(x)$, as desired. Hence, the 
continued fraction of $S_{n+1}(x)$ is determined by that of $S_{n}(x)$ by (7.4). Since the continued fraction of $S_{1}(x)$ is $\left[0, x,-g(x) x^{2}+(2 g(x)+1) x-g(x)-3, x+\right.$ $1, x-1]$, all of these continued fractions are specializable.

This completes the proof.

LEMma 7.10. Let $f(x)$ be a quadratic polynomial over $\mathbb{Z}$. Then the sum $\sum_{n=0}^{\infty} 1 / f^{n}(x)$ has a specializable continued fraction iff $f(x)=k x^{2}$ for some $k, f(x)=k x^{2}-x$ for some $k$, or $f(x)$ is one of $x^{2}-x+1, x^{2}-2 x+1$, $-x^{2}-x-1$, or $-x^{2}-2 x-1$.

(This lemma is proved the same way as the necessity of the conditions in Theorem 7.9. We omit the details. Essentially, one looks at partial sums and shows that they do not have specializable continued fractions, unless $f(x)$ belongs to one of the six cases of the lemma. Arguments like those used to prove the next lemma show that the beginnings of the continued fractions for these partial sums coincide with the beginning of the continued fraction for the entire series. In each case, the non-specializability of the continued fraction for the partial sum occurs near enough to its beginning to imply that the entire series cannot have a specializable continued fraction expansion.)

LEMma 7.11. Let $f(x)$ be a polynomial over $\mathbb{Z}$, of degree greater than 2. Then the partial sums of $\sum_{n=0}^{\infty} 1 / f^{n}(x)$ are convergents to its continued fraction.

Pro of. Suppose that $\operatorname{deg} f(x)=l$. Since the denominator of the partial sum $\sum_{n=0}^{k} 1 / f^{n}(x)$ clearly divides $f^{0}(x) f^{1}(x) \ldots f^{k}(x)$, it has degree at most $\left(l^{k+1}-1\right) /(l-1)$. Since $l \geq 3$, this is strictly less than $\frac{1}{2} \operatorname{deg} f^{k+1}(x)$.

Now note that if we define a valuation on $\mathbb{Q}(x)$ by $|a(x) / b(x)|=$ $2^{\operatorname{deg} a(x)-\operatorname{deg} b(x)}$, then it is easy to see that for $a, \varepsilon \in \mathbb{Q}(x),|a \varepsilon|<1$ implies $\varepsilon+1 / a=1 /\left(a+\varepsilon^{\prime}\right)$ with $\left|\varepsilon^{\prime}\right|=\left|\varepsilon a^{2}\right|$. From this, it follows immediately that $\sum_{n=0}^{k} 1 / f^{n}(x)$ is a convergent of $\sum_{n=0}^{k+1} 1 / f^{n}(x)$. This proves our lemma.

Combining the last three results yields the following theorem:

TheOREM 7.12. Let $f(x)$ be a polynomial over $\mathbb{Z}$, of degree greater than 1. Then $\sum_{n=0}^{\infty} 1 / f^{n}(x)$ has a specializable continued fraction if and only if $f(x)$ satisfies one of the fourteen congruences listed in the statement of Theorem 7.9.

Another interesting example is $f(x)=x^{2}+x-1$. The continued fraction in this case is not specializable, but no denominators greater than 2 occur. Doubling the continued fraction eliminates all but one of them. If a continued fraction is not specializable, but the coefficients of its partial quotients have denominators at most 2 , then we call it semi-specializable. Semi-specializable continued fractions are almost as interesting as special- 
izable ones, because it is fairly straightforward to transform a continued fraction whose partial quotients are halves of integers to a simple continued fraction. It might be interesting to find an analogue of Theorem 7.12 for semi-specializability, because it is conceivable that there would be a simpler or more interesting characterization.

8. Other forms of folding symmetry. In addition to folding symmetry, at least one other similar form of symmetry occurs in some continued fractions, this time in products rather than sums. Define $\vec{a}_{n}$ to be the word $a_{0}, a_{1}, \ldots, a_{n}$, and define $\overleftarrow{a_{n}}$ to be $a_{n}, a_{n-1}, \ldots, a_{0}$. Thus, $\vec{a}_{n}=a_{0}, \vec{w}_{n}$ This notation will be more convenient than the earlier notation.

Note that since $\left[\overleftarrow{a}_{n}\right]=p_{n} / p_{n-1}, \vec{a}_{n}=\overleftarrow{a}_{n}$ if and only if $p_{n-1}=q_{n}$. (To compute $\left[\overleftarrow{a_{n}}\right]$, note that $\left[\overleftarrow{a}_{n}\right]=-1 / f_{n}^{-1}(0)=p_{n} / p_{n-1}$.) We now use this to prove the following proposition:

Proposition 8.1. If $\vec{a}_{n}=\overleftarrow{a}_{n}$ then

$$
\left[\vec{a}_{n}, x, \overleftarrow{a}_{n}\right]=\frac{p_{n}}{q_{n}}\left(1+\frac{(-1)^{n}}{q_{n}\left(x p_{n}+2 q_{n}\right)+(-1)^{n-1}}\right)
$$

Proof. We have

$$
\left[\vec{a}_{n}, x, \overleftarrow{a}_{n}\right]=\frac{p_{n}\left(x+p_{n-1} / p_{n}\right)+p_{n-1}}{q_{n}\left(x+p_{n-1} / p_{n}\right)+q_{n-1}}
$$

Now if we apply the relation $p_{n} q_{n-1}=(-1)^{n-1}+q_{n} p_{n-1}$, we get

$$
\frac{p_{n}^{2} x+2 p_{n} p_{n-1}}{p_{n} q_{n} x+2 q_{n} p_{n-1}+(-1)^{n-1}},
$$

and substituting $p_{n-1}=q_{n}$ gives

$$
\frac{p_{n}^{2} x+2 p_{n} q_{n}}{p_{n} q_{n} x+2 q_{n}^{2}+(-1)^{n-1}},
$$

which is equal to

$$
\frac{p_{n}}{q_{n}}\left(1+\frac{(-1)^{n}}{q_{n}\left(x p_{n}+2 q_{n}\right)+(-1)^{n-1}}\right),
$$

as desired.

The symmetry appearing in Proposition 8.1 looks very much like 2-fold symmetry, but of course is not exactly the same. For lack of a better name, we call it duplicating symmetry.

Given the more restrictive hypotheses of Proposition 8.1 (compared to those of the Folding Lemma), one might not expect there to be any interesting applications. However, there are some. 
Products such as

$$
\prod_{n=0}^{\infty}\left(1+\frac{1}{x^{l^{n}}}\right)
$$

do not have $k$-fold or duplicating symmetry. (They do have interesting continued fraction expansions, which can be determined explicitly for $l$ even. For the details, see [7].) Surprisingly, the Chebyshev analogues

$$
\prod_{n=0}^{\infty}\left(1+\frac{1}{T_{l^{n}}(x)}\right)
$$

(where $T_{l}(x)$ is the $l$ th Chebyshev polynomial) have iterated duplicating symmetry, provided that $l \equiv 2(\bmod 4)$. We prove this in slightly greater generality.

Let $f(x)$ be a polynomial over $\mathbb{Z}$ of degree greater than 1 such that

$$
f(x) \equiv 2 x^{2}-1\left(\bmod x\left(x^{2}-1\right)\right)
$$

in $\mathbb{Z}[x]$. It is easy to prove by induction that $f^{i}(x)^{2}-1$ is divisible by $\left(f^{0}(x)+1\right)\left(f^{1}(x)+1\right) \ldots\left(f^{i}(x)+1\right)$. Also, note that $f^{i}(x)$ divides $f^{i+1}(x)+1$.

Now consider the product

$$
P_{n}=\prod_{i=0}^{n}\left(1+\frac{1}{f^{i}(x)}\right) .
$$

The remarks above show that its denominator is $f^{n}(x)$. We now show that it has duplicating symmetry. We can assume (by induction) that $P_{n-1}$ has a symmetric continued fraction, of odd length. (Note that $P_{0}=[1, x-1,1]$.) Now, in order to apply Proposition 8.1, we need only show that

$$
\frac{f\left(f^{n-1}(x)\right)-2 f^{n-1}(x)^{2}+1}{f^{n-1}(x)}
$$

is divisible by the numerator of $P_{n-1}$. Note that this is divisible by $f^{n-1}(x)^{2}$ -1 , and hence by $\left(f^{0}(x)+1\right)\left(f^{1}(x)+1\right) \ldots\left(f^{n-1}(x)+1\right)$. This implies that it is divisible by the numerator of $P_{n-1}$, and thus that $P_{n}$ has iterated duplicating symmetry. (Note that in the special case $f(x)=2 x^{2}-1$, we have $P_{\infty}=\sqrt{x^{2}-1} /(x-1)$. See [8].)

When $l \equiv 2(\bmod 4)$, the Chebyshev polynomial $T_{l}(x)$ satisfies the appropriate congruence. Since for all $a$ and $b, T_{a b}(x)=T_{a}\left(T_{b}(x)\right)$, it follows that

$$
\prod_{n=0}^{\infty}\left(1+\frac{1}{T_{l^{n}}(x)}\right)
$$

has iterated duplicating symmetry. Also, the partial quotients have integer coefficients, so the continued fraction is specializable. For $l \equiv 0(\bmod 4)$, there appears to be a semi-specializable continued fraction, which is somewhat, but not entirely, symmetric. For $l$ odd, things are more complicated. 
(In these cases, the continued fractions are almost certainly never specializable.)

9. Open questions and conjectures. Unfortunately, this paper fails to answer the following natural questions (among others):

(1) How do Theorems 7.9 and 7.12 generalize to products?

(2) Which of the sums studied in this paper have semi-specializable continued fractions? What about the products?

(3) There are a number of continued fraction expansions derived in this paper that are reminiscent of 2-fold or 3-fold symmetry. Are any of them special cases of more general phenomena (as 2-fold symmetry is a special case of $k$-fold symmetry)?

(4) Under what conditions is the sum of two specializable continued fractions still specializable? (At the end of Section 6, a number of cases in which this occurs are derived. However, the derivation does not seem to generalize in this direction.)

(5) Does Proposition 5.1 have any interesting or useful generalizations?

We can, however, conjecture some partial answers to these questions:

Conjecture 9.1. There exists a finite collection $\left\{\left(a_{i}(x), b_{i}(x)\right)\right\} \subset \mathbb{Z}[x]$ $\times \mathbb{Z}[x]$ such that each $b_{i}(x)$ divides $x^{2}\left(x^{2}-1\right)^{2}$, with the property that if $f(x) \in \mathbb{Z}[x]$ and $\operatorname{deg} f(x)>1$, then all of the partial products of $\prod_{n=0}^{\infty}(1+$ $\left.1 / f^{n}(x)\right)$ have specializable continued fractions if and only if for some $i$, $f(x) \equiv a_{i}(x)\left(\bmod b_{i}(x)\right)$.

It may be the case that $x^{2}\left(x^{2}-1\right)^{2}$ needs to be replaced by another polynomial, but even in that case, the (appropriately modified) conjecture is presumably true. Techniques similar to those used in the proof of Theorem 7.9 will probably work. One will presumably also arrive at a result analogous to Theorem 7.12.

It seems plausible that one can prove similar results for semi-specializability (in the case of products or that of sums).

ConjeCture 9.2. Let $l$ be a non-zero multiple of 4. Then

$$
\prod_{n=0}^{\infty}\left(1+\frac{1}{T_{l^{n}}(x)}\right)
$$

has a semi-specializable continued fraction expansion.

If true, this result probably arises from congruence conditions satisfied by the Chebyshev polynomials. The result about $x^{2}+x-1$ mentioned at the end of Section 7 may very well also generalize similarly. 
Acknowledgements. I am grateful to Jeffrey Shallit for reading a draft of this paper and providing helpful comments and suggestions.

\section{References}

[1] A. Blanchard et M. Mendès France, Symétrie et transcendance, Bull. Sci. Math. 106 (1982), 325-335.

[2] R. Graham, D. Knuth and O. Patashnik, Concrete Mathematics, AddisonWesley, Reading, 1989.

[3] G. H. Hardy and E. M. Wright, An Introduction to the Theory of Numbers, Oxford University Press, Oxford, 1979.

[4] M. Kmošek, Continued fraction expansion of some irrational numbers, Master's Thesis, Uniwersytet Warszawski, Warszawa, 1979 (in Polish).

[5] G. Köhler, Some more predictable continued fractions, Monatsh. Math. 89 (1980), $95-100$.

[6] M. Mendès France, Sur les fractions continues limitées, Acta Arith. 23 (1973), $207-215$.

[7] M. Mendès France and A. J. van der Poorten, Some explicit continued fraction expansions, Mathematika 38 (1991), 1-9.

[8] A. Ostrowski, Über einige Verallgemeinerungen des Eulerschen Produktes $\prod_{\nu=0}^{\infty}\left(1+x^{2^{\nu}}\right)=1 /(1-x)$, Verh. Naturf. Ges. Basel 2 (1929), 153-214.

[9] A. J. van der Poorten and J. Shallit, Folded continued fractions, J. Number Theory 40 (1992), 237-250.

[10] —, -, A specialised continued fraction, Canad. J. Math. 45 (1993), 1067-1079.

[11] J. Shallit, Simple continued fractions for some irrational numbers, J. Number Theory 11 (1979), 209-217.

[12] -, Simple continued fractions for some irrational numbers II, ibid. 14 (1982), 228231.

[13] J. Tamura, Explicit formulae for certain series representing quadratic irrationals, in: Number Theory and Combinatorics, J. Akiyama et al. (eds.), World Scientific, Singapore, 1985, 369-381.

[14] -, Symmetric continued fractions related to certain series, J. Number Theory 38 (1991), 251-264.

Department of Mathematics

Harvard University

Cambridge, Massachusetts 02138

U.S.A.

E-mail: cohn@math.harvard.edu 